\section{Eatist}

${ }^{1}$ Department of Thoracic Surgery, University Hospital Zurich, Zurich, Switzerland DDepartment of Pulmonary Medicine, University Hospital Zurich, Zurich, Switzerland ${ }^{3}$ Institute of Pathology and Molecular Pathology, University Hospital Zurich, Zurich, Switzerland

${ }^{4}$ Department of Radiology, University Hospital Zurich, Zurich, Switzerland

\section{Correspondence to} Dr Karina Brüstle, Department of Thoracic Surgery, University Hospital Zurich, Raemistrasse 100, Zurich 8091, Switzerland karina.bruestle@usz.ch

$\mathrm{KB}$ and $\mathrm{KZ}$ contributed equally.

Received 23 April 2017

Revised 8 July 2017 Accepted 7 August 2017

Published Online First 31 August 2017

\title{
Acute pulmonary embolism and multiple pulmonary nodules mimicking metastatic disease
}

\author{
Karina Brüstle, ${ }_{1}^{1}$ Katharina Zachariassen, ${ }^{2}$ Bart Vrugt, ${ }^{3}$ Thomas Frauenfelder, ${ }^{4}$ \\ Walter Weder, ${ }^{1}$ Daniel Franzen ${ }^{2}$
}

\section{CASE PRESENTATION}

A 62-year-old patient with a history of traumatic splenectomy after a paragliding accident and chronic hepatitis $\mathrm{C}$ presented to the emergency department with worsening dyspnoea and unintended weight loss of $8 \mathrm{~kg}$ in 2 months. He was a lifelong non-smoker and consumes alcohol on rare occasions. He also reported recent travels to Asia and South America for business affairs. On clinical examination, the patient was normotensive with a respiratory rate of $25 / \mathrm{min}$. Lung auscultation and the remainder of the physical examination were uneventful. Laboratory parameters showed increased inflammatory parameters (leucocyte count $14.7 \mathrm{~g} / \mathrm{L}, \mathrm{C}$ reactive protein $87 \mathrm{mg} / \mathrm{L})$, absolute eosinophilia $(4.73 \mathrm{~g} / \mathrm{L})$ and elevated $\mathrm{IgE}$ levels $(5170 \mathrm{kU} / \mathrm{L})$. A CT scan revealed acute segmental pulmonary embolism and multiple irregular pulmonary nodules (figure 1A). A bronchoscopy showed no airway abnormalities such as inflammation or tumour protrusion; however, bronchoalveolar lavage revealed eosinophilia.

\section{ASSESSMENT OF THE JUNIOR DOCTORS}

The radiological appearance mimicked metastatic disease. With multiple nodules, unintentional weight loss and a history of chronic hepatitis C, metastatic disease from hepatocellular carcinoma or progressive lung carcinoma was suspected. Furthermore, the initial workup showed a partly calcified liver lesion in the right lobe measuring $9 \times 10 \mathrm{~cm}$. A subsequent sonogram showed mild liver enlargement with homogenous parenchyma and an inhomogeneous, round liver lesion in segments V-VII. The corresponding CT showed a cystic lesion with scattered calcifications in the wall. With an extensive tropical travel history, common parasites as well as zoonotic diseases and fungal infection were included in the differential diagnoses.

\section{ASSESSMENT OF THE THORACIC SURGEON}

At the time of initial workup, a parasitic disease was included in the differential. However, given the extensive lung involvement and clinical symptoms mimicking malignancy, an underlying metastatic disease was the primary concern. At most, a concomitant parasitic infection was considered. With no definitive diagnosis after bronchoscopy, an interdisciplinary panel decided to perform thoracoscopic wedge resection of an exemplary pulmonary nodule for histopathological workup.

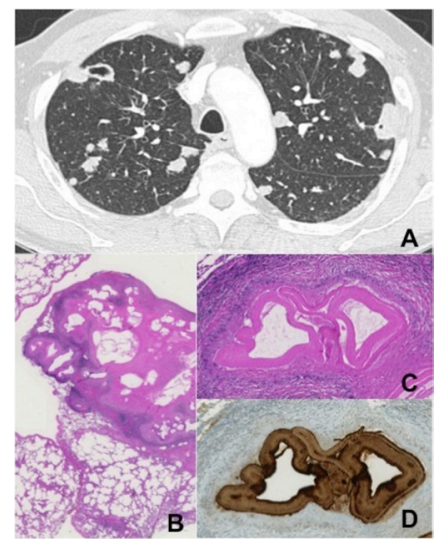

Figure 1 (A) CT thorax showing multiple irregular pulmonaly nodules. (B) H\&E staining: multifocal echinococcal cysts and granulomas forming an interface with adjacent alveolar tissue. (C) H\&E staining: detail showing cyst wall and pallisading histiocytes. (D) EM2 antigen staining: identifying echinococcus multilocularis.

\section{ASSESSMENT OF THE PATHOLOGIST}

Histology showed multifocal echinococcus cysts surrounded by pallisading histiocytes on H\&E staining (figure 1B). Adjacent to the cysts, granulomas are forming an interface with the adjacent alveolar tissue. In order to differentiate between the strains of echinococci, an EM2 antigen antibody stain was performed, which positively stained the laminar layer of the cyst, consistent with echinococcus multiocularis (figure1C).

\section{ASSESSMENT OF THE PULMONOLOGIST}

Echinococcus granulosus is the most frequent species observed in patients, followed by the rare but more virulent E. multilocularis. To complete their life cycle, parasites are surviving as larvae in hydatid cysts before inhabiting the intestines of canines as definitive hosts. Humans become aberrant hosts after egg ingestion by contaminated food or close contact with animal fur. The lung is the second most common organ affected with bilateral pulmonary disease occurring in 5\%-20\% of all pulmonary cases. Echinococcal parasites can settle into the lung via different pathways. After the ingested egg hatches, the gastrointestinal wall is penetrated, and the parasite gains access to the liver through portal circulation. Liver sinusoids are big enough to let small larvae pass through to access 
the hepatic vein, and subsequently the IVC, right heart and the lungs. ${ }^{1}$ In cases that show pulmonary invasion with sparing of the liver, it is suggested that echinococcal larvae gain access to the lymphatic route and bypass the liver via the thoracic duct. The evidence of diaphragmal involvement suggests yet another pathway where the liver dome itself is infiltrated, the diaphragm passed and access to the intercostal and parasternal lymphatics is gained. While several invasion pathways are used, the parasite is able to migrate without being detected by the host's immune system. The echinococcal cyst consists of three layers: an outer, adventitial layer (called pericyst) and a laminated membrane (called exocyst) that is impermeable for bacteria and acts as a shield against the attack of immunological cells. These two layers are the reason why eosinophilia, linked to parasitosis, is only evident in a quarter of all echinococcal cases. Other signs of parasitic infection such as leukocytosis or increased erythrocyte sedimentation rate are not consistently observed. The endocyst, or inner, germinative layer, consists of sterile fluid. In case of cyst rupture, this antigenic fluid is able to launch an anaphylactic reaction. In this case, prior splenectomy might be the reason for the unusual extent of pulmonary disease. Similar cases have been reported in patients with protozoic disease and, in our case, can be the explanation for multiple nodules in several lobes.

\section{ASSESSMENT OF THE RADIOLOGIST}

While imaging can be misleading, there are some radiological signs that are 'telltale signs' of echinococcal invasion. ${ }^{2}$ With the growth of the pulmonary cyst, air can leak in between the layers of pericyst and exocyst, leading to an 'air crescent sign' visible on CT scan. The progression of air leaking in between the cystic layers, a 'Cumbo' or 'onion peel sign' might be observed. Once the cyst ruptures and fluid evacuates, the endocyst collapses and floats on top of the remaining fluid, creating a 'waterlily sign'. If the cyst is eroding into a bronchiole, air is entering into the cyst giving the impression of a small, round and radiolucent 'air bubble sign'. Positron emission tomography (PET)-CT can show increased activity mostly with ruptured cysts. Serological testing can reveal echinococcal infection via ELISA or immunoblot. However, these can be false positive in helminthic infection, cancer and chronic immune disease.

\section{ASSESSMENT OF THE THORACIC SURGEON}

Therapeutic approach with pulmonary hydatidosis depends on the extent of the disease. With just a small number of cysts, presenting mostly peripherally, surgical management with wedge resection is the preferred treatment. Advantages of surgery are the removal of the entire cyst including germinative membrane. In case of close contact to the bronchial system and centrally located cysts, other treatments include decompression, instillation of agents and drainage via suction bronchoscopy. ${ }^{3}$ In case of fistula or more extensive but localised disease, a lobectomy is an alternative option. ${ }^{2}$ The principal treatment of ruptured cystic disease is surgery. Generally, in extensive cases like ours, a surgical approach is deemed too risky. In patients with contraindication for surgery or with multiorgan and extensive disease, medical with albendazole has been the gold standard for treatment. Albendazole shows better bioavailability compared with other drugs from the benzimidazole group. By interfering with glucose absorption within the parasite, it leads to degeneration of cell organelles and parasitic death. Although pulmonary hydatid disease is most of the times discovered via imaging and treatment such as thoracoscopic resection followed by medical therapy leads good results with low morbidity and mortality, some adverse events can be life threatening: cystic rupture into the pleural space can lead to pleural hydatidosis and secondary bacterial infection with subsequent pneumonia. ${ }^{4}$ Once the fluidic antigen comes in contact with the hosts immune system, an outburst of histamine release ensues leading to anaphylactic shock. Another serious adverse event is pulmonary embolisms after pulmonary cysts erode the bronchial system. Echinococcal cysts invade into pulmonary vessels and lead to mechanical obstruction. $^{5}$

\section{ASSESSMENT OF THE PULMONOLOGIST}

A therapy with albendazol $400 \mathrm{mg}$ twice daily was started. Furthermore, oral anticoagulation with rivaroxaban was established. At follow-up after 3 weeks, the patient reported less dyspnoea and stable laboratory parameters (leucocytes count: $7.41 \mathrm{~g} / \mathrm{L}, \mathrm{C}$ reactive protein: $62 \mathrm{mg} / \mathrm{L}$, absolute eosinophilia $(7.41 \mathrm{~g} / \mathrm{L})$. Treatment with albendazol was continued, and the patient was discharged home in improved condition.

\section{TAKE HOME MESSAGE}

Echinococcal disease is a zoonosis with increasing incidence each year. While most of the burden of over 20000 new cases per year are located in China, rising echinococcal infection in Europe is noted with possible correlation to increasing urbanisation of fox populations. After erosion of pulmonary cysts into the bronchial system, echinococcal cysts can invade the pulmonary vessels and lead to mechanical obstruction. This leads to a clinical presentation of acute pulmonary embolus. Echinococcal disease can present with extensive pulmonary involvement mimicking metastatic disease. Prior splenectomy might be a predisposition for the unusual extent of parasitic pulmonary disease. In extensive echinococcal disease, a surgical approach harbours increased risk for anaphylactic shock. Initial conservative treatment with albendazole is recommended. The nature of the echinococcal cyst layers shield the parasite against immunological response. Eosinophilia is only present in a quarter of echinococcal cases.

Contributors All authors contributed significantly to the submitted work: KB, KZ and DF drafted the first version of the manuscript. KB and KZ made an extensive literature search, while DF is the guarantor of the overall content. BV, TF and WW added relevant details including figures and important intellectual content. All authors were involved in the patient's care and gave final approval of the manuscript submitted.

Competing interests None declared.

Patient consent Obtained.

Provenance and peer review Not commissioned; externally peer reviewed.

(C) Article author(s) (or their employer(s) unless otherwise stated in the text of the article) 2018. All rights reserved. No commercial use is permitted unless otherwise expressly granted.

\section{REFERENCES}

1 Sarkar M, Pathania R, Jhobta A, et al. Cystic pulmonary hydatidosis. Lung India 2016;33:179-91.

2 Gulamhussein MA, Patrini D, Pararajasingham J, et al. Hepatopulmonary Fistula: a life threatening complication of hydatid disease. J Cardiothorac Surg 2015;10:1-5

3 Hejazi ME, Tekantapeh ST, Hasani S. A novel bronchoscope method (165 saline injection method) for complete extraction of ruptured pulmonary hydatid cyst. Clin Respir J 2016:1-5.

4 Cobanoglu U, Sayır F, Sehitoğlu A, et al. Therapeutic strategies for complications secondary to hydatid cyst rupture. Int J Clin Exp Med 2011;4:220-6.

5 Yuan Z, Maihesuti M, Wenya L, et al. Rare reason for pulmonary embolism: one case of pulmonary hydatid cyst and review of the literature. J Thromb Thrombolysis 2015;40:126-9. 